\title{
Low-mass lithium-rich AGB stars in the Galactic bulge: evidence for cool bottom processing? ${ }^{\star}$
}

\author{
S. Uttenthaler ${ }^{1}$, T. Lebzelter ${ }^{1}$, S. Palmerini ${ }^{2}$, M. Busso ${ }^{2}$, B. Aringer ${ }^{1}$, and M. T. Lederer ${ }^{1}$ \\ 1 Department of Astronomy, University of Vienna, Türkenschanzstraße 17, 1180 Vienna, Austria \\ e-mail: [uttenthaler; lebzelter; aringer; lederer]@astro.univie.ac.at \\ 2 Department of Physics, University of Perugia, via A. Pascoli 1, 06123 Perugia, Italy \\ e-mail: [palmerini; busso]@fisica.unipg.it
}

Received 14 May 2007 / Accepted 26 June 2007

ABSTRACT

\begin{abstract}
Context. The stellar production of the light element lithium is still a matter of debate.
Aims. We report the detection of low-mass, Li-rich Asymptotic Giant Branch (AGB) stars located in the Galactic bulge.

Methods. A homogeneous and well-selected sample of low mass, oxygen-rich AGB stars in the Galactic bulge has been searched for the absorption lines of Li. Using spectral synthesis techniques, we determine from high resolution UVES/VLT spectra the Li abundance in four out of 27 sample stars, and an upper limit for the remaining stars.

Results. Two stars in our sample have a solar Li abundance or above; these stars seem to be a novelty, since they do not show any s-element enhancement. Two more stars have a Li abundance slightly below solar; these stars do show s-element enhancement in their spectra. Different scenarios which lead to an increased Li surface abundance in AGB stars are discussed.

Conclusions. Of the different enrichment scenarios presented, Cool Bottom Processing (CBP) is the most likely one for the Li-rich objects identified here. Self-enrichment by Hot Bottom Burning (HBB) seems very unlikely as all Li-rich stars are below the HBB mass limit. Also, the ingestion of a low mass companion into the stars' envelope is unlikely because the associated additional effects are lacking. Mass transfer from a former massive binary companion is a possible scenario, if the companion produced little s-process elements. A simple theoretical estimation for the Li abundance due to CBP is presented and compared to the observed values.
\end{abstract}

Key words. nuclear reactions, nucleosynthesis abundances - stars: AGB and post-AGB - stars: evolution - stars: abundances

\section{Introduction}

The AGB represents the final nuclear-burning phase of stellar evolution for stars with initial masses between $\sim 0.8$ and $8.0 M_{\odot}$. The internal structure consists of an electron-degenerate carbonoxygen core which is the remainder of He-core and -shell burning, a He-rich shell on top of which $\mathrm{H}$ is burning in a shell, and a deep outer convective envelope. For a comprehensive review of AGB evolution see e.g. Habing \& Olofsson (2003). In the most luminous part of the AGB, the behaviour of a star is characterised by the so-called thermal pulses (TP), thermal instabilities of the He shell accompanied by changes in luminosity, temperature, period, and internal structure (Busso et al. 1999; Herwig 2005). Before a star reaches the AGB its previous evolution has significantly changed its surface abundance pattern. For instance, the ${ }^{12} \mathrm{C} /{ }^{13} \mathrm{C}$ ratio has been considerably lowered from its initial (solar) value by the first dredge-up on the lower RGB. A remarkable effect is the diminishing of Li. This element is destroyed in stars once it is mixed to layers where the temperature exceeds $3.0 \times 10^{6} \mathrm{~K}$ (Bodenheimer 1965). Due to its fragility, lithium is an important diagnostic tool for stellar evolution (Rebolo 1991), but also has a high significance in the determination of cosmological parameters (Spite \& Spite 1982; Korn et al. 2006).

* Based on observations at the Very Large Telescope of the European Southern Observatory, Cerro Paranal/Chile under Program 65.L-0317(A, B).
In intermediate mass $\left(\gtrsim 4-8 M_{\odot}\right)$ thermally pulsing AGB (TP-AGB) stars, the outer convective envelope penetrates into the H-burning shell, thus nuclear-burning occurs partly under convective conditions. This process is generally known as Hot Bottom Burning (HBB; Iben 1973). Under these conditions, ${ }^{7} \mathrm{Li}$ is produced via the so-called Cameron-Fowler or ${ }^{7} \mathrm{Be}$-transport mechanism $\left({ }^{3} \mathrm{He}(\alpha, \gamma){ }^{7} \mathrm{Be}\left(\mathrm{e}^{-}, v\right){ }^{7} \mathrm{Li}\right.$; Cameron \& Fowler 1971). The first detection of massive Li-rich AGB stars was reported by Smith \& Lambert (1989, 1990), and confirmed later to be in agreement with HBB models (Smith et al. 1995). Most recently, García-Hernández et al. (2007) presented Li abundance measurements of massive Galactic AGB stars.

Different types of evolved stars with Li not fulfilling the criteria for HBB are known in the literature. Li-rich K-type stars are presented in de la Reza et al. (1997). A Li-rich red giant (probably RGB) has been reported by Smith et al. (1999). Only a few low luminosity S-type (Smith et al. 1995; Van Eck et al. 1998) and C-type (Abia et al. 1991) Li-rich AGB stars are known.

In this paper we present the discovery of Li-rich, low mass M-type AGB stars in the Galactic bulge and discuss different mechanisms for the Li enrichment.

\section{Observations and analysis}

Spectra of the present sample of O-rich (M-type) Galactic bulge AGB stars have been originally obtained for a search for the radioactive element technetium (Tc) in these stars, which is an 
indicator of recent or ongoing third dredge-up (3DUP). The observed spectra and their analysis with respect to the occurrence of lines of Tc has been presented in Uttenthaler et al. (2007, hereafter called Tc-paper) in detail.

The homogeneous and well-selected sample of long-period variables in the Palomar Groningen field No. 3 (PG3), located in the outer Galactic bulge (Galactic latitude $b \sim-10^{\circ}$ ), has been collected from Wesselink (1987). Twenty-seven targets out of this sample were observed with the UVES spectrograph at ESO's VLT in July 2000. Besides the blue arm $(377-490 \mathrm{~nm})$ that was used to search for the occurrence of Tc, also the red arm (667-847 nm and 865-1050 nm) was covered by the observations. The signal-to-noise ratio (SNR) - as given by the ESO pipeline - achieved in the spectral region of the Li resonance doublet varies quite strongly between 14 and 240 per pixel, although most stars (including the ones reported here to show Li) have an SNR greater than 30 .

From the occurrence of Tc in four out of the 27 sample stars, and from their periods and luminosities, a minimum main sequence mass of $\sim 1.5 M_{\odot}$ for the Tc-rich stars was estimated. This has an effect on the inferred bulge age, since stars of that mass cannot be much older than 3-4 Gyr. This is in contrast to what is found from studies of other stellar types in the bulge, but well in line with a number of works on bulge AGB stars (see Tc-paper).

To determine the stellar parameters of our stars for the abundance analysis, we calculated a small grid of hydrostatic model atmospheres. The atmospheres are based on the MARCS code (see Tc-paper for details). The grid covered the following stellar parameter space: $T_{\mathrm{eff}}=2600-3600 \mathrm{~K}$ in steps of $100 \mathrm{~K}$, $\log (g)=-0.5$ and $0.0,[\mathrm{M} / \mathrm{H}]=-0.5,0.0$, and +0.2 . Ti was enhanced by 0.2 dex in the models (McWilliam \& Rich 1994). Due to their rather limited effect on the appearance of the spectra the mass and the $\mathrm{C} / \mathrm{O}$ ratio were fixed to the values $1.0 M_{\odot}$ and 0.48 , respectively. The microturbulent velocity was set to $3 \mathrm{~km} \mathrm{~s}^{-1}$.

Synthetic spectra based on these model atmospheres were calculated for the two wavelength ranges $668-674 \mathrm{~nm}$ and $700-710 \mathrm{~nm}$, respectively. The first piece covers (besides the Li line) the TiO $\gamma(1,0) \mathrm{Rc}$ and $\gamma(1,0) \mathrm{Rb}$ band heads, the second piece covers the $\mathrm{TiO} \gamma(0,0) \mathrm{Ra}$ and $\gamma(0,0) \mathrm{Rb}$ band heads. These band heads are rather sensitive to $T_{\text {eff. }}$. The synthetic spectra were convolved with a Gaussian to reduce the resolution to the value of the observed spectra $(R=50000)$, and an additional macro-turbulence of $4 \mathrm{~km} \mathrm{~s}^{-1}$ was added in the convolution.

A $\chi^{2}$ minimisation method over the mentioned spectral ranges was applied to find the main parameters of the sample stars. As comparison between the two spectral regions used for the fit showed, the temperature yielding the best fit differed substantially between the two regions in many of the observed stars. On average, the temperature derived from the region around the Li-line was lower by about $100 \mathrm{~K}$ then the temperature derived from the second wavelength range. We suspect the reason for this is an underestimation of the $\mathrm{TiO} \gamma(1,0) \mathrm{Rc}$ and $\gamma(1,0) \mathrm{Rb}$ band head strength in the line list used (Schwenke 1998). A similar problem has been reported in Reiners (2005) for other band heads of TiO. Thus, it seems possible that several $\mathrm{TiO}$ bands are incorrect in their strength, and a revision of the current line list is desirable.

Spectral synthesis calculations were applied in order to identify stars which show signs of Li line absorption. For these calculations we chose the model yielding the lowest $\chi^{2}$ value in the spectral region around the $\mathrm{Li}$ line, despite the obvious discrepancy regarding the derived temperature. The reason for this is that the background $\mathrm{TiO}$ absorption is thus modelled as well as possible. The four stars with a positive Li detection could
Table 1. Sample stars with positive Li detection. The bolometric magnitude is corrected for depth effects within the bulge.

\begin{tabular}{lrrccr}
\hline \hline Name & $M_{\text {bol }}$ & $P$ (days) & $\log \epsilon(\mathrm{Li})$ & $\Delta \log \epsilon(\mathrm{Li})$ & Tc? \\
\hline M 45 & -4.52 & 271.02 & 2.0 & 0.5 & no \\
M 794 & -4.76 & 303.54 & 1.1 & 0.4 & no \\
M 1147 & -5.28 & 395.63 & 0.8 & 0.4 & yes \\
M 1347 & -5.43 & 426.60 & 0.8 & 0.4 & yes \\
\hline
\end{tabular}

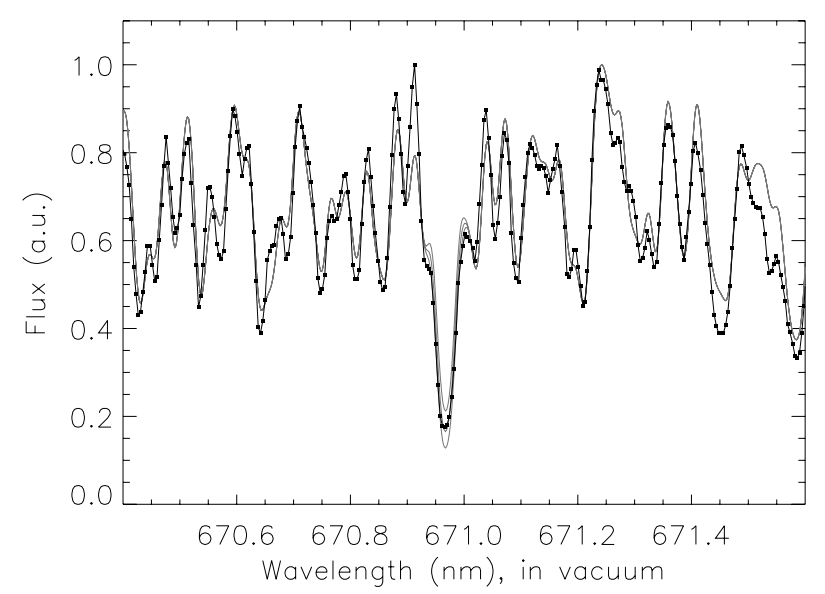

Fig. 1. Observed spectrum of the most Li-rich sample star M45 around the $671 \mathrm{~nm} \mathrm{Li}$ line together with the synthetic spectrum (grey line) used for the $\mathrm{Li}$ abundance determination, assuming $\mathrm{Li}$ abundances of $\log \epsilon(\mathrm{Li})=1.8, \log \epsilon(\mathrm{Li})=2.0$, and $\log \epsilon(\mathrm{Li})=2.2$.

all be fitted with a single atmospheric model. This model has $T_{\text {eff }}=3000 \mathrm{~K}, \log (g)=0.0$, and $[\mathrm{M} / \mathrm{H}]=0.0$.

We assessed the content of s-process elements in our sample stars by investigating the strength of the $\mathrm{ZrO}$ band head at $462 \mathrm{~nm}$ with respect to the $\mathrm{TiO} \gamma(0,0) \mathrm{Ra}$ band head at $705.6 \mathrm{~nm}$. Also, atomic line strengths of the s-elements $\mathrm{Sr}$, $\mathrm{Y}, \mathrm{Zr}, \mathrm{Ba}, \mathrm{La}, \mathrm{Ce}, \mathrm{Nd}$, and $\mathrm{Sm}$ in the range 400-460 nm were inspected. Only the previously identified Tc-rich stars showed an increased strength of these features.

\section{Discussion}

For four stars a non-negligible $\mathrm{Li}$ abundance has to be assumed in order to give satisfying fits to the observed spectrum. Their determined Li abundances and bolometric magnitudes (corrected for the bulge depth scatter, see the Tc-paper) are summarised in Table 1. The error on the $\mathrm{Li}$ abundance given in the fifth column was estimated by varying the temperature of the atmospheric model by $\pm 100 \mathrm{~K}$. For the other stars, an upper limit to the $\mathrm{Li}$ abundance was estimated. For the hotter stars $(\sim 3400 \mathrm{~K}$, Semi-regular variables), this upper limit is around $\log \epsilon(\mathrm{Li})=$ 0.6 , for the cooler stars $(\sim 3000 \mathrm{~K}$, Mira variables $)$, it is around $\log \epsilon(\mathrm{Li})=0.1^{1}$. Actually, excellent fits for the spectra of the Li-poor stars can be achieved by fully neglecting $\mathrm{Li}$ in the spectral synthesis. Figure 1 shows the observed spectrum of M 45, the most Li-rich star in our sample, along with synthetic spectra with differing Li abundance.

The first thing we note is that the bolometric magnitudes of the stars listed in Table 1 are considerably below the luminosity expected for Li-enrichment due to HBB. (In models

1 Throughout the paper, the $\mathrm{Li}$ abundance is given on the scale $\log \epsilon(\mathrm{Li})=\log N(\mathrm{Li}) / N(\mathrm{H})+12$. The solar photospheric $\mathrm{Li}$ abundance on this scale is $\log \epsilon(\mathrm{Li})=1.1$. 
with rather extreme convective overshoot, HBB may start already at luminosities as low as $M_{\text {bol }} \simeq-5$. 0 (Karakas 2003), but Li-enrichment becomes observable only at $M_{\text {bol }} \simeq-6$. 0 (Smith et al. 1995; Vanture et al. 2007).) Regarding the bolometric magnitudes of these stars, and also age considerations of the Galactic bulge, the HBB scenario is very unlikely to account for the observed $\mathrm{Li}$ abundances.

A few stars with Li enrichment but $M_{\text {bol }}$ below -6 . 0 have been reported in the literature. Smith et al. (1995) identify a low luminosity, S-type star with very high Li abundance in the SMC (HV 1645). They state a bolometric magnitude of -4.98 for that star. Abia et al. (1991) derives $M_{\text {bol }}=-5$. 0 for the C-rich star WX Cyg. Three S-stars below the HBB limit, namely NO Aur, $\pi^{1}$ Gru and HR Peg, are listed by Van Eck et al. (1998).

Another remarkable thing about M 45 and M 794 in our sample is the absence of Tc-lines from their spectra. Both stars do not show any other signs of s-process enhancement either, while M 1147 and M 1347 do show Tc and s-process enhancement. Among a sample of Galactic MS- and S-type stars Vanture et al. (2007) found three groups of stars with respect to the $\mathrm{Li}$ and Tc abundance: those without $\mathrm{Tc}$ and $\mathrm{Li}$, interpreted as the result of mass transfer from a more massive companion; those with $\mathrm{Tc}$ and $\mathrm{Li}$, which are intermediate-mass stars producing Li through HBB; and those with Tc but no Li, which are thought to be AGB stars below the HBB mass limit. One star of their sample, V441 Cyg, shows no Tc but is enriched in Li. This star as well as the low luminosity S- and C-type stars with Li found before were interpreted as due to Cool Bottom Processing (see Sect. 3.3).

The stars M 45 and M 794 in our sample seem to be indeed a novelty, since they are the first AGB stars detected with a considerable $\mathrm{Li}$ overabundance, but no indications for a third dredgeup. From their variability and their location in the bulge CMD both stars are certainly on the AGB. In the following we will discuss these two stars in the light of the various Li-enrichment scenarios.

\subsection{Enrichment by massive binary companion}

The Li-rich stars might have received Li-rich matter by wind accretion from a close massive $\left(M \gtrsim 4 M_{\odot}\right)$ binary companion which experienced HBB and might be a white dwarf now. One might think that a possible mass transfer would have left its signature not only in the presence of $\mathrm{Li}$, but also in the abundance of s-process elements, since such a massive star can be expected to have undergone s-processing and 3DUP during its TP-AGB phase. This mass transfer would not have shown up in our search for Tc due to the short life time of this element. As mentioned above, our Li-only stars show also no enhancement in the stable s-process elements. However, Li enrichment is expected to occur in early AGB phases (Karakas 2003), when s-elements might still be absent. Moreover, intermediate mass stars might never become very rich in s-elements, as their prevailing ${ }^{22} \mathrm{Ne}$ source never builds up large neutron exposures, and the massive envelope dilutes extensively any new material dredged-up. Indeed, García-Hernández et al. (2007) did not find enhanced $\mathrm{ZrO}$ band and atomic $\mathrm{Zr}$ I, Nd II, and Ba II line strengths in their sample of massive Galactic Li-rich AGB stars. This implies only a low s-process level in these stars. In contrast, Smith et al. (1995) find a considerable level of s-process enrichment for their Li-rich AGB stars in the Magellanic Clouds. The difference in the behaviour of massive AGB stars located in the Magellanic Clouds and in the Milky Way galaxy might be a metallicity effect, but conclusive interpretation is not yet possible. It is not known how the bulge fits into this picture.

A possible WD companion may be detectable via an excess flux in the $U$ or $B$ band. Mean $B$ and $R$ band values for the two most Li-rich stars, M 45 and M 794, were found in the literature, but they did not turn out to be conspicuously blue in $(B-R)$.

In conclusion, the massive binary companion hypothesis is not fully convincing, but cannot be ruled out on the basis of current observations.

\subsection{Accretion of a (sub-)stellar companion}

Siess \& Livio (1999) investigated the response of the structure and abundances of an AGB star to the accretion ("swallowing") of a (sub-)stellar companion. One result of their considerations is that the effect on the Li abundance might only be detectable if a considerable mass $\left(\gtrsim 0.1 M_{\odot}\right)$ is accreted to the envelope of the AGB star. Other expected effects of a brown dwarf accretion include the increase of the mass loss rate and a spin-up of the envelope. These would be detectable as an IR excess and the rotational broadening of spectral lines, respectively. M45 and M794, the two most Li-rich stars in our sample were not detected with IRAS, while M 1147 and M 1347 were. For the latter two mass loss rates of the order of $10^{-6.6} M_{\odot} / \mathrm{yr}$ have been derived in our Tc-paper. Since these two stars are also the brightest and longest period stars in our sample, this mass loss rate can be expected due to normal evolution. Also, Siess \& Livio (1999) estimate the fraction of stars with a low-mass companion which may be accreted during the evolution of the primary to 4-8 percent. However, $15 \%$ of our sample stars show Li. We conclude that the sub-stellar accretion scenario is unlikely to explain the Li abundances observed in our sample stars.

\subsection{Cool bottom processing}

It is now known (see e.g. Kraft 1994; Charbonnel 2004) that the radiative layers below the convective envelope in evolved, low-mass red giants are the site of slow mixing phenomena, in addition to the convective dredge-up episodes. Such phenomena have been variously called "deep mixing", or "extended mixing", or Cool Bottom Processing (CBP), although this last name is relatively recent (Wasserburg et al. 1995). The chemical stratification of a radiative layer hampers mass motions; hence, mixing can take place only after the H-burning shell has erased any chemical discontinuity left by core $\mathrm{H}$ burning and by the first dredge-up. When this occurs, the luminosity shows a bump, after which abundance changes related to extra-mixing begin to appear.

As to the physical origin of the extended mixing phenomena, very recently a number of hypotheses have been presented, at least for RGB stars. Eggleton et al. (2006), on the basis of a 3D simulation, found that Rayleigh-Taylor instabilities below the convective envelope can develop due to the inversion of the mean molecular weight gradient induced by ${ }^{3} \mathrm{He}$ burning. Alternatively, Charbonnel \& Zahn (2007) suggested that the double-diffusive mechanism called "thermohaline instability" should be at play. In principle, both these phenomena might occur also on the AGB, though detailed models have not been presented. Finally, Busso et al. (2007) explore the possibility that circulation of partially processed matter can be accounted for by magnetic buoyancy induced by a stellar dynamo operating on the RGB and on the AGB. 
Independently of the still uncertain physical cause, we know that the best established effect is a shift in the carbon isotopic mix (Gilroy \& Brown 1991), decreasing from the typical value of 25-30 left by the first dredge-up to 10-15 for Population I stars and down to 4 for Population II objects. Other consequences include $\mathrm{N}$ enrichment and ${ }^{18} \mathrm{O}$ depletion (Charbonnel \& Do Nascimiento 1998). Similar CBP episodes can occur later, during the thermally pulsing AGB stages, where again the sub-adiabatic zone below the envelope has a homogeneous molecular weight. Several authors (Boothroyd \& Sackmann 1999; Charbonnel \& Balachandran 2000) studied the possibility that ${ }^{7} \mathrm{Li}$ is affected by circulation phenomena. In particular it was noted that, on the RGB, Li enrichment is limited to phases close to the bump itself, so that it was suggested that Li production accompanies the early onset of extra-mixing (Charbonnel \& Balachandran 2000).

Let us illustrate what is found in stellar codes, using a model star of initially $1.5 M_{\odot}$, with a Population I composition $([\mathrm{Fe} / \mathrm{H}]=-0.3$; Busso et al. 2003; Straniero et al. 2003). Here production of ${ }^{7} \mathrm{Li}$ derives entirely from (bound or free) electron captures on ${ }^{7} \mathrm{Be}$. The synthesis of ${ }^{7} \mathrm{Be}$ can be followed through the competition of production and destruction:

$\frac{\mathrm{d} N\left({ }^{7} \mathrm{Be}\right)}{\mathrm{d} t}=N(3) N(4) \lambda_{3,4}-N\left({ }^{7} \mathrm{Be}\right) N(\mathrm{p}) \lambda_{7, \mathrm{p}}-N\left({ }^{7} \mathrm{Be}\right) \lambda_{7, \mathrm{e}^{-}}$

where $N(3), N(4), N(\mathrm{p})$ are the number abundances of ${ }^{3} \mathrm{He},{ }^{4} \mathrm{He}$ and protons, and $\lambda$ indicates the reaction rate. In high temperature regions $\left(T>2 \times 10^{7} \mathrm{~K}\right){ }^{7} \mathrm{Be}$ is completely ionised so that the contribution to $\mathrm{e}^{-}$captures coming from bound electrons is suppressed. Here the synthesis of ${ }^{8} \mathrm{~B}$ through $\mathrm{p}$ captures is efficient and prevails.

In the mentioned stellar model the mass dependence of the rates for ${ }^{7} \mathrm{Be}$ production $\left[\lambda\left({ }^{3} \mathrm{He}+{ }^{4} \mathrm{He}\right)\right]$ and for its destruction through proton $\left[\lambda\left({ }^{7} \mathrm{Be}+\mathrm{p}^{+}\right)\right]$or electron $\left[\lambda\left({ }^{7} \mathrm{Be}+\mathrm{e}^{-}\right)\right]$captures is shown in Fig. 2, for the case where p-captures occur without mass circulation. In this case ${ }^{7} \mathrm{Be}$ reaches an equilibrium concentration $N_{7}^{\mathrm{e}}$ that depends on the ${ }^{3} \mathrm{He}$ abundance maintained by $\mathrm{H}$ burning, and that remains pretty low. In the same hot zones, and down to $T \geq 3-4 \times 10^{6} \mathrm{~K}$, any ${ }^{7} \mathrm{Li}$ remaining captures protons and is very efficiently destroyed.

The same qualitative behaviour is maintained in presence of circulation phenomena too slow to save most of the produced ${ }^{7} \mathrm{Be}$ into regions of low $T$ (where it decays into ${ }^{7} \mathrm{Li}$ ). Actually, any mixing phenomenon occurring at sufficiently low rates (a few $10^{-8} M_{\odot} / \mathrm{yr}$ characterises Red Giants) would destroy $\mathrm{Li}$ in the envelope, carrying it to hot regions where it is burnt, while its replenishment through ${ }^{7} \mathrm{Be}$ saved at low $T$ would remain too small to compensate.

In order to achieve a net and durable Li enhancement in the envelope it is required that a mixing mechanism of the type inferred by Cameron \& Fowler (1971) carries ${ }^{7} \mathrm{Be}$ rather rapidly out of the high-temperature zone, typically to $<3 \times 10^{6} \mathrm{~K}$. On the one hand in such low- $T$ regions the ionisation equilibrium of ${ }^{7} \mathrm{Be}$ favours the presence of bound electrons, from which $\mathrm{e}^{-}$captures are increased. These dominate over p-captures (that are essentially shut off). On the other hand, in these layers any ${ }^{7} \mathrm{Li}$ produced would survive and the total inventory of ${ }^{7} \mathrm{Li}$ to be carried to the envelope can be largely increased. We can have an estimate for the maximum ${ }^{7} \mathrm{Li}$ production if we consider the pure production of ${ }^{7} \mathrm{Be}$ in Eq. (1), without destruction by p-captures, as if it were quickly carried to cool zones. Its derivative in time is shown in Fig. 2 as $\left(\mathrm{d} N_{7} / \mathrm{d} t\right)_{\mathrm{p}}$. The integral of that curve over the production region and over a period of one year provides the

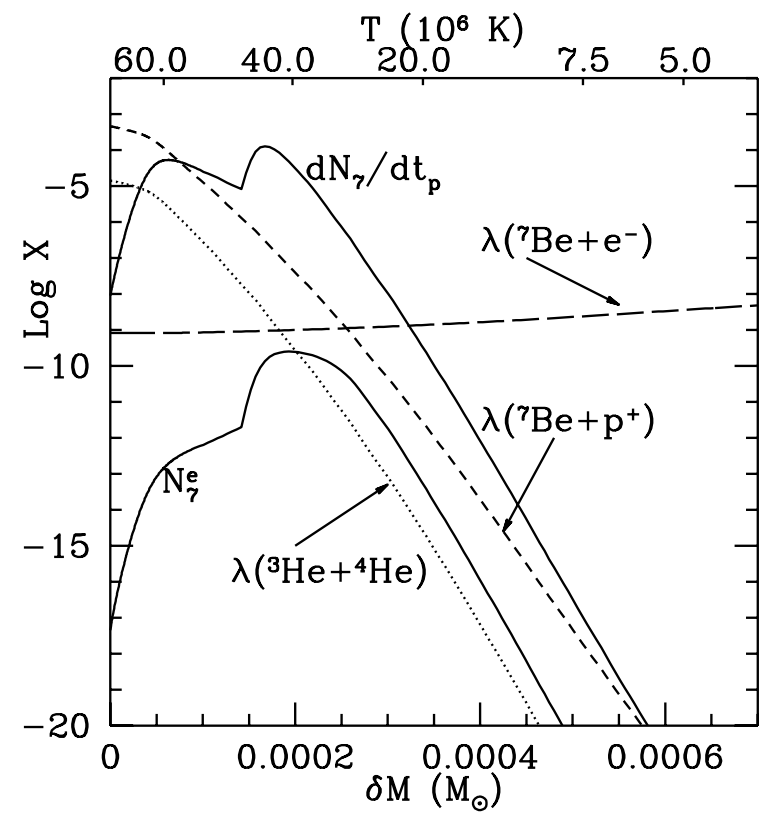

Fig. 2. Rates for ${ }^{7}$ Be production and destruction in the radiative layers below the convective envelope (dotted and dashed lines). The abscissa shows the distance in mass from the deepest layers affected by CBP, so that the convective envelope is at the right, outside the plot area. Also plotted is the ${ }^{7} \mathrm{Be}$ equilibrium abundance $\left(N_{7}^{\mathrm{e}}\right)$ and its production rate (without destruction) if an infinite ${ }^{3} \mathrm{He}$ reservoir is available $\left(\mathrm{d} N_{7} / \mathrm{d} t\right)_{\mathrm{p}}$. This mimics the supply from the ${ }^{3} \mathrm{He}$ present in the envelope in case of extended mixing.

maximum mass of Li that can be produced per year, amounting to $5.65 \times 10^{-13} M_{\odot} / y r$. In order to have the envelope (of about $\left.1 M_{\odot}\right)$ enriched up to a mass fraction $X(\mathrm{Li})=2 \times 10^{-8}$ ( $\simeq 10$ times solar) we need to mix the produced $\mathrm{Li}$ at a rate $\dot{M}>3 \times 10^{-5} M_{\odot} / y r$, which is a rather fast circulation rate. Such high mixing rates have been so far suggested to occur only in rather extreme AGB cases, in order to explain some isotopic and elemental shifts there inferred from measurements on pre-solar grains of AGB origin. They would produce isotopic and elemental abundance changes (including Li) hardly distinguishable from hot bottom burning effects (Nollett et al. 2003), but for much lower stellar masses. In previous calculations with complete models (e.g. Boothroyd \& Sackmann 1999) Li production was obtained only adopting very fast circulation rates $\left(\dot{M} \simeq 10^{-4} M_{\odot} / \mathrm{yr}\right)$. This result is in line with our rather rough estimate for the lower $\dot{M}$ limit $\left(3 \times 10^{-5} M_{\odot} / \mathrm{yr}\right)$.

\section{Conclusions}

We conclude that the $\mathrm{Li}$ abundance measured for M45 is probably to be ascribed to the onset of rather extreme CBP phenomena on the AGB. This interpretation would be in line with suggestions by Nollett et al. (2003) and is the first hint for deep mixing coming from an M-type AGB star. As a consequence of the efficient mixing, we expect the ${ }^{12} \mathrm{C} /{ }^{13} \mathrm{C}$ ratio to be close to the equilibrium value of 3.5 . A remarkable ${ }^{14} \mathrm{~N}$ enhancement at the surface would occur only if zones of relatively high temperature $(\log T>7.4)$ were involved in the mixing episodes. This enhancement is instead expected in any case if HBB was at play. We leave these predictions for a subsequent verification of the consistency of our explanation. At this stage we can only consider CBP as the most likely hypothesis, but we cannot derive too strong conclusions against the other interpretations, 


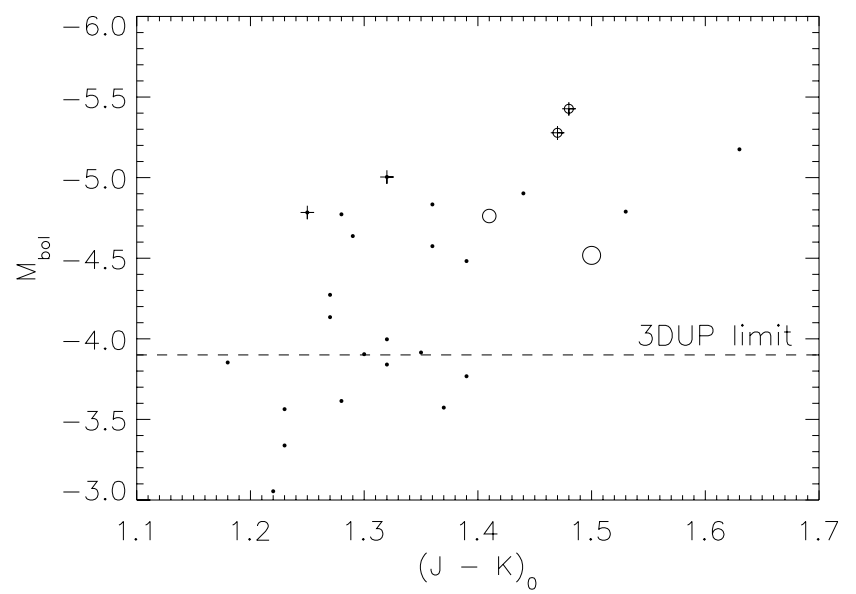

Fig. 3. Bolometric magnitude versus $(J-K)_{0}$ of our sample stars. Open circles are stars with positive Li detection, the size of the circle corresponds to the determined abundance of Li. Symbols with a cross denote stars with positive Tc detection. The dashed horizontal line indicates the estimated lower luminosity limit for 3DUP.

in particular the binary wind accretion hypothesis, on the basis of current observations.

Our findings also suggest that the conditions for CBP and those for the third dredge up are independent. CBP is expected to be favoured at low masses, while below a certain mass limit (depending on the metallicity) dredge-up of s-processed material does not occur. The Li-rich stars M 45 and M 794 should therefore be TP-AGB objects of really low mass $\left(M \leq 1.3-1.5 M_{\odot}\right)$, where very efficient CBP can occur, while the core is not massive enough to drive dredge-up.

Despite the lack of a physical explanation for the origin of CBP, a few hints can be found from the investigation of the present homogeneous sample. Judging from Fig. 3, the luminosities and temperatures of the Li-rich stars without Tc are similar to the Tc-rich stars. Also, the pulsation periods are comparable: The Li-only stars M 45 and M 794 have periods of around or just slightly below $300 \mathrm{~d}$, which is about the minimum period of the Tc stars.

M 45 and M794 have Li abundances higher than the two Tc stars M 1147 and M 1347, and M 626 and S942, the two remaining $\mathrm{Tc}$ stars, do not show $\mathrm{Li}$ at all. It is quite thinkable that in the mass range of our sample stars Li production via CBP sets in before 3DUP occurs, or for masses slightly lower than necessary to drive dredge-up. We note that the radiative layers below the convective envelope are sub-adiabatic, chemically homogeneous zones where no molecular weight barrier hampers the circulation of matter, so that the onset of CBP should be much easier than formal dredge-up.
In any case, since not all of the stars with pulsation periods around or above $300 \mathrm{~d}$ and luminosities comparable to the Li-rich stars do show this element, very special conditions have to be met in order for CBP to work at the rather extreme rates necessary. Which these conditions are remains a question to be answered.

Acknowledgements. T.L. and S.U. acknowledge funding by the Austrian Science Fund FWF under the project P18171-N02, and BA acknowledges funding by the FWF under project P19503-N13. M.T.L. has been supported by the Austrian Academy of Sciences (DOC-programme). S.P. and M.B. acknowledge funding from MURST under contract PRIN2006-022731. We also acknowledge J. Hron (Vienna) for devising the bulge UVES-project and for carrying out the observations.

\section{References}

Abia, C., Boffin, H. M. J., Isern, J., \& Rebolo, R. 1991, A\&A, 245, L1 Bodenheimer, P. 1965, ApJ, 142, 451

Boothroyd, A. I., \& Sackmann, I.-J. 1999, ApJ, 510, 232

Busso, M., Gallino, R., \& Wasserburg, G. J. 1999, ARA\&A, 37, 239

Busso, M., Gallino, R., \& Wasserburg, G. J. 2003, PASA, 20, 356

Busso, M., Wasserburg, G. J., Nollett, K. M., \& Calandra, A. 2007, ApJ, submitted

Cameron, A. G. W., \& Fowler, W. A. 1971, ApJ, 164, 111

Charbonnel, C. 2004, in Origin and Evolution of the Elements (Cambridge, UK: Cambridge Univ. Press), ed. A. McWilliam, \& M. Rauch, 60

Charbonnel, C., \& Balachandran, S. C. 2000, A\&A, 359, 563

Charbonnel, C., \& Do Nascimiento, J. D. 1998, A\&A, 336, 915

Charbonnel, C., \& Zahn, J.-P. 2007, A\&A, 467, 15

de la Reza, R., Drake, N. A., \& da Silva, L. 1997, ApJ, 482, L77

Eggleton, P. P., Dearborn, D. S. P., \& Lattanzio, J. C. 2006, Science, 314, 1580

García-Hernández, D. A., García-Lario, P., Plez, B., et al. 2007, A\&A, 462, 711

Gilroy, K. K., \& Brown, J. A. 1991, ApJ, 371, 578

Habing, H. J., \& Olofsson, H. 2003, Asymptotic Giant Branch Stars, Astron.

Astrophys. Library (New York: Springer)

Herwig, F. 2005, ARAA, 43, 435

Iben, I. 1973, ApJ, 185, 209

Karakas, A. 2003, Ph.D. Thesis, Monash University, Australia

Korn, A., Grundahl, F., Richard, O., et al. 2006, Nature, 442, 657

Kraft, R. P. 1994, PASP, 106, 553

Mcwilliam, A., \& Rich, R. M. 1994, ApJS, 91, 749

Nollett, M. N., Busso, M., \& Wasserburg, G. J. 2003, ApJ, 582, 1036

Rebolo, R. 1991, IAUS, 145, 85

Reiners, A. 2005, AN, 326, 930

Schwenke, D. 1998, Faraday Discuss., 109, 321

Siess, L., \& Livio, M. 1999, MNRAS, 304, 925

Smith, V. V., \& Lambert, D. L. 1989, ApJ, 345, L75

Smith, V. V., \& Lambert, D. L. 1990, ApJ, 361, L69

Smith, V. V., Plez, B., Lambert, D. L., \& Lubowich, D. A. 1995, ApJ, 441, 735

Smith, V. V., Shetrone, M. D., \& Keane, M. J. 1999, ApJ, 516, L73

Spite, M., \& Spite, F. 1982, Nature, 297, 483

Straniero, O., Domínguez, I., Cristallo, S., \& Gallino, R. 2003, PASA, 20, 389

Uttenthaler, S., Hron, J., Lebzelter, T., et al. 2007, A\&A, 463, 251; Tc-Paper

Van Eck, S., Jorissen, A., Udry, S., Mayor, M., Pernier, B. 1998, A\&A, 329, 971

Vanture, A. D., Smith, V. V., Lutz, J., et al. 2007, PASP, 119, 147

Wasserburg, G. J., Boothroyd A. I., \& Sackmann, I.-J. 1995, ApJ, 447, L37

Wesselink, Th. J. H. 1987, Ph.D. Thesis, Catholic University of Nijmengen, The Netherlands 